\title{
EFFECTS OF HEALTH EDUCATION BASED ON OMAHA SYSTEM ON ANXIETY, DEPRESSION AND SELF-MANAGEMENT ABILITY OF PRIMIPARA
}

\author{
Qiong Pan', Ying Huang ${ }^{2} \&$ Baofeng Duan ${ }^{3}$ \\ ${ }^{I}$ Yunnan Xinxing Occupations Institute, Kunming, Yunnan, China \\ ${ }^{2}$ Department of Nursing, Yan'an Hospital of Kunming City, Kunming, Yunnan, China \\ ${ }^{3}$ Department of Nursing, Affiliated Hospital of Yunnan University, Kunming, Yunnan, China \\ received: 25.6.2021; \\ revised: 22.9.2021; \\ accepted: 4.10 .2021
}

SUMMARY

Background: Primiparas are prone to tension, anxiety, depression and other emotions due to their lack of experience related to delivery and fear of the unknown during pregnancy. Meanwhile, their self-management ability decreases because of their failure to immediately adapt to the transformation of their roles in a short time. A nursing model was constructed in this study based on the combination of the Omaha System with PBL health education to examine the effect of this model on the anxiety, depression, quality of life and self-management ability of primiparas.

Subjects and methods: From January to December 2020, 170 primiparas were recruited as volunteers in this study by means of voluntary registration. They were randomly divided into the observation group and the control group, with 85 members in each group. The intervention lasted 6 weeks. SDS, SAS, WHOQOL-BREF scale and ESCA scale were used to compare the scores of the two groups before and after the intervention.

Results: (1) After the intervention, the changes of the observation group in self-management ability and its four dimensions are higher than those of the control group, with the differences being statistically significant $(P<0.05)$. (2) After the intervention, the changes of the observation group in quality of life and its four dimensions are higher than those of the control group, with the differences being statistically significant $(P<0.05)$. (3) The changes of the observation group in anxiety and depression are higher than those of the control group. Specifically, the change of the observation group in anxiety before and after the intervention is $6.40 \pm 5.61$, in comparison with $2.67 \pm 3.71$ in the control group; the change of the observation group in depression before and after the intervention is $9.07 \pm 8.42$, in comparison with $3.19 \pm 7.06$ in the control group.

Conclusion: the new nursing method proposed in this study effectively improves the self-management ability and quality of life of primiparas, significantly reduces their anxiety and depression, has a high application value in obstetric nursing and also provides a new idea for the implementation of scientific and efficient nursing for primiparas.

Key words: The Omaha System - health education - primiparas - anxiety and depression

$$
* * * * *
$$

\section{INTRODUCTION}

Pregnancy is a special physiological stage throughout a woman's life. During this period, the woman would experience significant physiological changes and psychological stress, probably thereby suffering physical and psychological harm (Coll et al. 2017) and subsequently having emotional problems such as anxiety and depression (Melo \& Arzeno 2016). Noticeably, depression is the most common mental illness arising from pregnancy (Ayele et al. 2016). It has been found that nearly $20-30 \%$ of pregnant women suffer from depression (Mossie et al. 2017). Female depression mostly occurs during pregnancy and postpartum, especially postpartum. Postpartum depression refers to a mix of a series of depressive symptoms in a pregnant woman during the stage of puerperium. Its clinical manifestations include downbeat mood, reduced interest, poor appetite, tension and paranoia. Some pregnant women with serious symptoms may even commit suicide and infanticide ( $\mathrm{Lu}$ et al. 2020). Obviously, postpartum depression not only undermines the physical and mental health of pregnant women, but also poses a great threat to their infants and families. As revealed in a research, postpartum depression has an incidence rate of $10-20 \%$, generally occurring within the first 2 weeks postpartum and gradually worsening in the $4^{\text {th }}-6^{\text {th }}$ weeks postpartum (Woody et al. 2017). Those patients whose postpartum depression lasts 6 months or longer account for $25-50 \%$ of the total number of patients with postpartum depression (Ye et al. 2016). Apart from depression, anxiety is also one of the most universal adverse psychological reactions in pregnant women. Relevant statistical data shows that the incidence of anxiety in pregnant women is $20-60 \%$ (Shi et al. 2019). Depression and anxiety symptoms always coexist (Sartorius et al. 1996) and boost each other over time (McElroy et al. 2018). Anxiety during pregnancy predicts the occurrence of postpartum depression, while prenatal depression also predicts the incidence of postpartum anxiety (Skouteris et al. 2009). Compared with multiparae, primiparas have no experience related to delivery, are afraid of the unknown during pregnancy, and are prone to tension, 
anxiety, depression and other negative emotions (Nakamura et al. 2020). Their postpartum self-efficacy is extremely low because they generally fail to immediately adapt to their new role within a short time. Meanwhile, the decrease of their self-efficacy may also lead to a decline in their self-management ability. According to relevant reports, those with suicidal tendency account for $3.8 \%$ of all the patients with postpartum depression, of which $1.1 \%$ belong to the group with high risk of suicide. Hence, it is particularly important to choose feasible and efficient nursing measures to minimize or eliminate postpartum depression (Zhao \& Jing 2019).

At present, most nurses in China have to engage in busy nursing work due to the serious shortage of frontline clinical nurses. Due to the absence of a scientific nursing thinking model of applying nursing procedures in clinical work under this context, clinical nurses, in the process of implementing nursing procedures, cannot make appropriate nursing diagnosis in response to the problems appearing in patients. As a simplified nursing procedure, the Omaha System puts forward nursing problems according to the symptoms and signs of patients, and then provides a guide through the intervention system for nurses to formulate nursing measures. In this way, it can help nurses make correct nursing diagnosis, highly enhancing the pertinence of the nursing plan and the accuracy of the nursing measures implemented. The Omaha System (OS) is on the list of the 12 nursing standardization languages recognized by the American Nurses Association (ANA), and consist of three parts, including the problem classification system, the intervention classification system and the effect evaluation system (Maxim et al. 2014). It can comprehensively evaluate patients' condition and nursing intervention, quantifies nursing problems and intervention effects, and thus effectively guides clinical nursing practice. In a study where the Omaha System was applied to 41 hospitalized patients with chronic obstructive pulmonary disease, a total of 268 nursing problems were found, with each patient having 6.54 nursing problems on average. These results indicate that in the process of nursing hospitalized patients with chronic obstructive pulmonary disease, the application of the Omaha System contributes to the discovery of multiple complex nursing problems other than those related to the respiratory system, gives operation guidelines for nurses to master the occurrence law of nursing problems during hospitalization, and also establishes the foundation for providing patients with satisfactory high-quality nursing (Xiao 2018). Beňová et al. used the Omaha System in patients with chronic gastrointestinal diseases to intervene in them from four aspects, including environment, psychology, physiology, and health. After comparing the initial results to the final results, there was significant improvement in the knowledge and health condition regarding individual problems.
Accordingly, the Omaha System can effectively improve the health status of patients with chronic diseases and play a positive role both in the continuous improvement of nursing quality and the supervision of clinical work (Beňová et al. 2016). It was claimed that the Omaha System can record the implementation details of clinical intervention, including what happened, when and where it happened, the cause of its occurrence, and all aspects of patients, so that researchers can quickly identify the complex nursing problems of patients (Martin et al. 2011). Although the Omaha System has been partially adopted in the field of nursing in China, Chinese nursing workers have neither widely recognized and utilized relevant research findings nor frequently engaged in practical applications, which is not conducive to the development of nursing work and the progress of the nursing discipline. Therefore, it is of great significance to apply the Omaha System to primiparas during their pregnancy.

Health education aims to establish correct health concepts in patients. When it comes to obstetric nursing, the widely used health education methods are oral education, classroom intended for pregnant women, and personalized health education. However, the single model of health education and rich contents of health education usually prevent pregnant women from remembering or taking the initiative. This type of unidirectional and non-targeted knowledge transfer does not reinforce the compliance of pregnant women, so that it is not conducive to postpartum rehabilitation (Wang 2019). PBL health education (PBL: ProblemBased Learning) is a heuristic education model oriented to diseases, targeting patients, guided by nurses, and constructed with all learning contents conveyed in the form of problems. Specifically, PBL health education is advantageously featured with high pertinence, full consideration of the real needs and individualized interests of patients in terms of content and form, mobilization of patients' awareness to take active participation and elimination of patients' psychological resistance ( $\mathrm{Su}$ 2016). Although it has been shown in previous studies that PBL health education has a certain effect on the negative psychology of primiparas, little academic attention has been paid to implementation of PBL health education for primiparas based on the Omaha nursing model.

Therefore, PBL pregnancy health education was applied based on the Omaha System in this study, with the focus on problem-solving and the mobilization of patients' active participation. Medical workers were asked to provide targeted relevant knowledge and skill support for primiparas. The objective is to investigate the impact of this new health education model on the depression, anxiety and self-management ability primiparas, in order to provide a new perspective for obstetric nursing health education and offer useful reference for the nursing of other diseases. 


\section{SUBJECTS AND METHODS}

\section{Participants}

170 primiparas from Yan'an Hospital of Kunming City, The Second People's Hospital Of Yunnan Province, Yunnan Province, China were included as the participants of this study by means of voluntary registration from January to December 2020. Inclusion criteria:

- those aged below 35 years old;

- primiparas;

- those who began to seek consultation and examination in this hospital after 3 months of pregnancy;

- those with normal results of pelvic measurement and fetal position examination;

- those who planned to deliver in this hospital;

- those who were informed of the study and willing to sign the informed consent of the study;

- the study met relevant standards approved by the ethics committee.

Exclusion criteria:

- those with multiple pregnancy;

- those with severe hearing, vision or communication impairment;

- those with intellectual or mental diseases;

- those with severe physical diseases;

- those with severe pregnancy complications;

- those suffered depression and anxiety in the first 3 months of pregnancy as a result of other major life events.

The participants were randomly divided into the control group and the observation group, with $85 \mathrm{mem}$ bers in each group. As shown in Table 1, there is no significant difference between the two groups in age, gestational weeks, relationship with family member, education level and family economic level $(\mathrm{P}>0.05)$, which means that they are comparable. Before this study, the purpose, main operation methods and precautions of this study were explained to all the participants and their guardians. All the scales were filled in anonymously. This study has been reviewed and approved by the medical ethics committee of the hospital, with the informed consent from the participants and their families.

\section{Methods}

The control group was given routine intervention and health education: during hospitalization, the participants were given routine intervention such as admission introduction, ward environment, pregnancy health care and monitoring, provided with the pregnancy knowledge manual uniformly compiled by the hospital, exposed to health education pregnancy knowledge through ward bulletin board and video broadcast, and given necessary psychological support. In addition to accepting the same routine intervention and health education available in the control group, the observation group was given pregnancy nursing based on the combination of the Omaha System nursing model and PBL health education. The intervention lasted 6 weeks. The specific methods are as follows:

\section{Problem classification system}

After the primipara was admitted to the hospital, she was asked to give information about her delivery, mood, parenting and family situation. A detailed evaluation of her nursing problems was conducted according to the problem classification table in the Omaha System nursing model, to summarize the current problems of the primipara from four aspects: health, physiology, psychology and environment. In view of the summarized problems, relevant nursing improvement measures were searched, selected, and sorted out to formulate an overall nursing intervention scheme.

\section{Intervention system}

It could be divided into cognitive intervention, psychological intervention and family \& social support.

- Cognitive intervention: A Wechat group or QQ group was established for primiparas, where knowledge regarding self-care and emotional disorders during pregnancy was regularly delivered every day, to inform them of the important role of positive mood during pregnancy in postpartum rehabilitation. In addition to the sharing of some short videos and image materials about skills and precautions in postpartum care and neonatal feeding in the QQ group, timely responses were given to relevant questions raised by each primipara. These efforts were expected to enrich their understanding of postpartum emotional disorders, improve their ability of self-care and neonatal feeding, enable them to calmly solve many problems encountered in postpartum life, and help them maintain peace of mind.

- Psychological intervention: efforts were made to identify the psychological state of primiparas through online chat, perceive their anxiety, depression and other negative emotions in time, understand the causes of their negative emotions, use positive wordings to comfort them, encourage them to talk about their unhappiness or confusion, give appropriate suggestions according to the causes of their negative emotions, and guide them to relieve negative emotions through listening to soothing music, taking a deep breath or reading favorite books.

- Family \& social support: Nursing workers were required to effectively convey the harm of negative mood in primiparas to their husband, parents and other family members, emphasize the importance of family and social support for the mood of primiparas, and ask their family members to do a good job in relevant cooperation and bring primiparas positive psychological support through spending more time to accompany and care them in daily life, sharing the 
responsibility of newborn feeding, and taking the initiative to deal with family affairs, so that primiparas can have a harmonious and loving family environment. In addition, primiparas were also guided to have more communication with other primiparas in the same period, talk to each other, release psychological their pressure, exchange parenting experience, and seek a sense of group belonging and external identity.

\section{Evaluation system}

Nursing workers evaluated the effectiveness of the implemented intervention scheme every week through online video call and telephone follow-up, find out deficiencies, adjust the intervention methods and perfect the nursing scheme.

The intervention lasted 6 weeks. In particular, questionnaire survey was used to investigate the problems of primiparas and their corresponding needs respectively before the intervention and in the third week of the intervention. Then, related problems were evaluated and summarized to finally form a comprehensive and detailed PBL question database. Nursing workers carried out PBL health education in class in response to the collected problems, about $1 \mathrm{~h}$ each time. Targeted health education was delivered in combination with the evaluated differences of primiparas in their personality, acceptance ability and needs. During the implementation of nursing measures in this study, nursing workers actively communicated with primiparas to trace their mastery of health knowledge, carried out key education when necessary, and organized family members to learn together till primiparas grasped relevant knowledge, timely encouraged primiparas to take active participation and mobilized their initiative as much as possible. Effects were measured through the following scales, including Self-rating Anxiety Scale (SAS) (Zung 1971) and Self-rating Depression Scale (SDS) (Zung 1965) used to analyze the mental health status of all patients before the intervention (during enrollment) and after the intervention (before delivery), Evaluation of Self-care Ability (ESCA) (Kearney \& Fleischer 1979, Wang et al. 2014) Scale used to evaluate and compare the selfmanagement ability of patients, and WHOQOL-BREF Scale (Wang \& Wang 2013) used to evaluate the quality of life of patients.

\section{Measuring Tools}

\section{Self-rating Anxiety Scale (SAS) (Zung 1971)}

The patients were asked to have a self-rating of their anxiety before and after the intervention according to the SAS rating standard. This scale compiled by Zung has been widely used in the clinical diagnosis and treatment of diseases such as diseases in the nervous system, and has turned out to have high reliability and validity. The scale contains 20 items, with each item being scored at 4 levels. 1 point means no or occasional; 2 points means sometimes; 3 points means frequent; 4 points means always. The final score is the sum of the scores of the 20 items multiplied by 1.25 . A higher score suggests that the symptoms are more serious. The score $<50$ points represent no anxiety; 50-59 represents mild anxiety; 60-69 represents moderate anxiety; the score $>69$ points represents severe anxiety.

\section{Self-rating Depression Scale (SDS) (Zung 1965)}

The patients were asked to have a self-rating of their depression before and after the intervention according to the SDS rating standard. The rating method is the same as that of SAS. The final score is the sum of the scores of the 20 items multiplied by 1.25. A higher score suggests that the symptoms are more serious. The score $<50$ points represent no depression; 50-59 represents mild depression; 60-69 represents moderate depression; the score $>69$ points represents severe depression.

\section{WHOQOL-BREF Scale (Wang \& Wang 2013)}

It is one of the effective simplified forms used to conduct clinical evaluation of patients'quality of life, with high reliability and validity. This scale can evaluate pregnant women from various aspects, including psychological state, physiological state, environment and social relations, with each aspect being converted into a percentage. A higher score suggests that the patient has better quality of life.

\section{Evaluation of Self-care Ability (ESCA) Scale}

ESCA was designed and developed by American scholars Kearney and Fleischer (Kearney \& Fleischer 1979) in 1979 according to Orem's Self-care Theory. The scale contains 43 items in four dimensions, namely, self-care skills, self-care responsibility, self-concept and health knowledge level. Thereafter, Wang et al. (Wang et al. 2014) tested the reliability and validity of the scale and concluded that the Chinese version of ESCA has good reliability and validity since its Cranach's $\alpha$ coefficientis 0.88 and its test-retest reliability is 0.62 . This scale was adopted herein to value the self-care ability of the patients before and after the six-week intervention. The scale has 43 items in total in four dimensions: self-concept, health knowledge level, selfcare responsibility and self-care skills. It has a total score of 172, with each item scored from 0 and 4 . A higher score indicates that the self-care ability is higher.

\section{Statistical Analysis}

The data were analyzed with SPSS19.0 statistical software. The quantitative data in line with normal distribution were expressed as mean \pm standard deviation. The comparison between two groups of data was conducted using independent sample t-test (comparison between groups) or paired t-test (comparison before and after intervention). The qualitative data were expressed as number of cases. The comparison between two groups of ordered classified data was conducted using Wilcoxon rank sum test, with $\mathrm{P}<0.05$ being statistically significant. 


\section{RESULTS}

\section{Demographic data}

170 participants (volunteers) were included in the study and randomly divided into the control group and the observation group, with 85 members in each group. A total of 164 participants finally completed the intervention and follow-up, including 81 in the observation group (4 lost in follow-up) and 83 in the control group (2 lost in follow-up). As shown in Table 1, there is no significant difference between the two groups in age, gestational weeks, relationship with family members, education level and family economic level $(\mathrm{P}>0.05)$, indicating that they are comparable.

\section{Comparison between the two groups in change in self-management ability before and after intervention}

It can be seen from table 2 that there is no significant difference between the observation group and the control group in self-management ability and its four dimensions before the intervention $(\mathrm{P}>0.05)$. After the intervention, there are increases in both groups, with the differences with the levels before the intervention being statistically significant $(\mathrm{P}<0.001)$. From the changes of the two groups before and after the intervention, the changes of the observation group in selfmanagement ability and its four dimensions are greater than those of the control group, with the differences being statistically significant $(\mathrm{P}<0.05)$, suggesting better intervention effects on the observation group than the control group.

\section{Comparison between the two groups in change in quality of life before and after intervention}

It can be seen from table 3 that there is no significant difference between the observation group and the control group in quality of life and its four dimensions before the intervention $(\mathrm{P}>0.05)$. After the intervention, there are increases in both groups, with the differences with the levels before the intervention being statistically significant $(\mathrm{P}<0.001)$. From the changes of the two groups before and after the intervention, the changes of the observation group in quality of life and its four dimensions are greater than those of the control group, with the differences being statistically significant $(\mathrm{P}<0.05)$, suggesting better intervention effects on the observation group than the control group.

\section{Comparison between the two groups in changes in anxiety and depression before and after intervention}

It can be seen from table 4 that there is no significant difference between the observation group and the control group in anxiety and depression before the intervention $(\mathrm{P}>0.05)$. After the intervention, there are decreases in the anxiety and depression scores of both groups, with the differences with the levels before the intervention being statistically significant $(\mathrm{P}<0.001)$. From the changes of the two groups before and after the intervention, the changes of the observation group in anxiety and depression scores are greater than those of the control group, with the differences being statistically significant $(\mathrm{P}<0.05)$, suggesting better intervention effects on the observation group than the control group.

Table 1. Comparison of demographic data between the observation group and the control group

\begin{tabular}{lcccc}
\hline & Observation group $(\mathrm{n}=81)$ & Control group $(\mathrm{n}=83)$ & Statistical value & $\mathrm{P}$ \\
\hline Age & $27.10 \pm 3.62$ & $26.92 \pm 4.42$ & $\mathrm{t}=0.290$ & 0.772 \\
Gestational weeks & 22 & & & \\
28 weeks & 25 & 27 & $\mathrm{Z}=0.969$ & 0.333 \\
29 weeks & 34 & 29 & & \\
30 weeks & & & & \\
Relationship with family members & 19 & 23 & $\mathrm{Z}=0.698$ & 0.485 \\
$\quad$ Very good & 23 & 25 & & \\
Good & 20 & 17 & & \\
General & 12 & 11 & \\
Bad & 7 & 7 & \\
$\quad$ Very bad & 12 & 13 & \\
Education level & 14 & 12 & & \\
Junior school or below & 19 & 17 & & \\
High or technical school & 23 & 28 & & \\
technical college & 13 & 13 & & \\
Undergraduate & & & & \\
Master degree or above & 18 & 19 & & \\
Family economic level & 20 & 25 & & \\
Very good & 23 & 19 & & \\
Good & 14 & 12 & & \\
General & 6 & 8 & & \\
Bad & & & & \\
Very bad & & & & \\
\hline
\end{tabular}


Table 2. Comparison between the two groups in change in self-management ability before and after intervention

\begin{tabular}{|c|c|c|c|c|}
\hline $\begin{array}{l}\text { Index } \\
\text { Group }\end{array}$ & $\begin{array}{c}\text { Before } \\
\text { intervention }\end{array}$ & $\begin{array}{c}\text { After } \\
\text { intervention }\end{array}$ & $\begin{array}{l}\text { Difference before and } \\
\text { after intervention }\end{array}$ & $\begin{array}{l}\mathrm{P} \text { value of inter-group } \\
\text { comparison }\end{array}$ \\
\hline \multicolumn{5}{|l|}{ Health knowledge level } \\
\hline Experimental group $(n=83)$ & $31.7 \pm 7.56$ & $42.9 \pm 8.01$ & $11.2 \pm 3.4$ & $<0.001$ \\
\hline Control group $(n=83)$ & $31.81 \pm 6.35$ & $38.77 \pm 7.37$ & $6.96 \pm 3.88$ & $<0.001$ \\
\hline $\mathrm{t}$ & 0.095 & 3.436 & 7.427 & \\
\hline $\mathrm{P}$ & 0.924 & 0.001 & $<0.001$ & \\
\hline \multicolumn{5}{|l|}{ Self responsibility } \\
\hline Experimental group $(n=83)$ & $18.35 \pm 5.61$ & $24.17 \pm 5.94$ & $5.83 \pm 2.44$ & $<0.001$ \\
\hline Control group $(n=83)$ & $18.01 \pm 5.26$ & $21.45 \pm 5.46$ & $3.43 \pm 1.42$ & $<0.001$ \\
\hline $\mathrm{t}$ & 0.393 & 3.062 & 7.698 & \\
\hline $\mathrm{P}$ & 0.695 & 0.003 & $<0.001$ & \\
\hline \multicolumn{5}{|l|}{ Self-nursing skills } \\
\hline Experimental group $(n=83)$ & $28.52 \pm 7.78$ & $37.64 \pm 7.94$ & $9.12 \pm 3.58$ & $<0.001$ \\
\hline Control group $(n=83)$ & $27.9 \pm 8.75$ & $30.76 \pm 8.76$ & $2.86 \pm 2.57$ & $<0.001$ \\
\hline $\mathrm{t}$ & 0.475 & 5.269 & 12.895 & \\
\hline $\mathrm{P}$ & 0.635 & $<0.001$ & $<0.001$ & \\
\hline \multicolumn{5}{|l|}{ Self-concept } \\
\hline Experimental group $(n=83)$ & $18.79 \pm 7.67$ & $26.53 \pm 6.92$ & $7.74 \pm 3.5$ & $<0.001$ \\
\hline Control group $(n=83)$ & $18.99 \pm 9.1$ & $21.81 \pm 8.65$ & $2.82 \pm 2.56$ & $<0.001$ \\
\hline $\mathrm{t}$ & 0.150 & 3.857 & 10.294 & \\
\hline $\mathrm{P}$ & 0.881 & $<0.001$ & $<0.001$ & \\
\hline
\end{tabular}

Table 3. Comparison between the two groups in change in quality of life before and after intervention

\begin{tabular}{|c|c|c|c|c|}
\hline $\begin{array}{l}\text { Index } \\
\text { Group }\end{array}$ & $\begin{array}{c}\text { Before } \\
\text { intervention }\end{array}$ & $\begin{array}{c}\text { After } \\
\text { intervention }\end{array}$ & $\begin{array}{l}\text { Difference before and } \\
\text { after intervention }\end{array}$ & $\begin{array}{l}P \text { value of inter-group } \\
\text { comparison }\end{array}$ \\
\hline \multicolumn{5}{|l|}{ Psychological state } \\
\hline Experimental group $(n=83)$ & $56.49 \pm 7.99$ & $66.15 \pm 8.42$ & $9.65 \pm 2.52$ & $<0.001$ \\
\hline Control group $(\mathrm{n}=83)$ & $56.08 \pm 8.37$ & $62.17 \pm 8.46$ & $6.08 \pm 2.63$ & $<0.001$ \\
\hline $\mathrm{t}$ & 0.320 & 3.019 & 8.866 & \\
\hline $\mathrm{P}$ & 0.749 & 0.003 & $<0.001$ & \\
\hline \multicolumn{5}{|l|}{ Physiological state } \\
\hline Experimental group $(\mathrm{n}=83)$ & $50.54 \pm 8.12$ & $60.25 \pm 7.83$ & $9.70 \pm 4.01$ & $<0.001$ \\
\hline Control group $(n=83)$ & $50.02 \pm 8.13$ & $55.34 \pm 8.18$ & $5.31 \pm 5.01$ & $<0.001$ \\
\hline $\mathrm{t}$ & 0.409 & 3.926 & 6.183 & \\
\hline $\mathrm{P}$ & 0.683 & $<0.001$ & $<0.001$ & \\
\hline \multicolumn{5}{|l|}{ Environment } \\
\hline Experimental group $(\mathrm{n}=83)$ & $51.89 \pm 8.31$ & $61.36 \pm 8.41$ & $9.47 \pm 2.89$ & $<0.001$ \\
\hline Control group $(n=83)$ & $50.96 \pm 8.39$ & $56.28 \pm 8.32$ & $5.31 \pm 2.58$ & $<0.001$ \\
\hline $\mathrm{t}$ & 0.709 & 3.888 & 9.720 & \\
\hline $\mathrm{P}$ & 0.479 & $<0.001$ & $<0.001$ & \\
\hline \multicolumn{5}{|l|}{ Social relations } \\
\hline Experimental group $(n=83)$ & $61.17 \pm 8.23$ & $75.22 \pm 8.2$ & $14.12 \pm 2.72$ & $<0.001$ \\
\hline Control group $(n=83)$ & $61.19 \pm 8.45$ & $68.01 \pm 8.38$ & $6.82 \pm 2.62$ & $<0.001$ \\
\hline $\mathrm{t}$ & 0.015 & 5.552 & 17.514 & \\
\hline $\mathrm{P}$ & 0.988 & $<0.001$ & $<0.001$ & \\
\hline
\end{tabular}

Table 4. Comparison between the two groups in changes in anxiety and depression before and after intervention

\begin{tabular}{lcccc}
\hline Index & $\begin{array}{c}\text { Before } \\
\text { Group }\end{array}$ & $\begin{array}{c}\text { After } \\
\text { intervention }\end{array}$ & $\begin{array}{c}\text { Difference before and } \\
\text { after intervention }\end{array}$ & $\begin{array}{c}\text { P value of inter-group } \\
\text { comparison }\end{array}$ \\
\hline Anxiety & & & & $<0.001$ \\
$\quad$ Experimental group $(\mathrm{n}=83)$ & $58.90 \pm 8.37$ & $52.51 \pm 9.91$ & $6.40 \pm 5.61$ & $<0.001$ \\
Control group (n=83) & $59.08 \pm 8.02$ & $56.41 \pm 8.79$ & $2.67 \pm 3.71$ & 5.021 \\
t & 0.143 & 2.671 & $<0.001$ & $<0.001$ \\
P & 0.886 & 0.008 & & $<0.001$ \\
Depression & & & $6.40 \pm 5.61$ & \\
Experimental group $(\mathrm{n}=83)$ & $58.90 \pm 8.37$ & $52.51 \pm 9.91$ & $2.67 \pm 3.71$ & \\
Control group (n=83) & $59.08 \pm 8.02$ & $56.41 \pm 8.79$ & 5.021 & \\
t & 0.143 & 2.671 & $<0.001$ & \\
P & 0.886 & 0.008 & & \\
\hline
\end{tabular}




\section{DISCUSSION}

In recent years, the increasing incidence of postpartum depression and anxiety has a great adverse impact on pregnant women's physical and mental health, neonatal development, families and even society ( $\mathrm{Lu}$ et al. 2020). Hence, it is pressing to monitor and prevent postpartum anxiety and depression. Although pregnancy and delivery are a natural biological process, it is actually a stress event for pregnant women, especially primiparas. Due to lack of delivery-related experience, primiparas are more likely to adopt negative coping styles to face this stress event (Li et al. 2021). It has been demonstrated that negative coping styles such as self-blame, evasion and giving up are not conducive to the treatment of health problems in primiparas, and their negative and pessimistic attitude towards the future easily leads to the occurrence of negative emotions such as anxiety and depression (Seda \& Erker 2021). Therefore, it is of great significance to choose effective nursing measures to alleviate the anxiety and depression of primiparas and improve their self-care ability and quality of life.

According to the results of this study, there is no significant difference between the observation group and the control group in anxiety and depression before the intervention. After a period of intervention, there are significant decreases in the anxiety and depression levels of the two groups, but the changes of the observation group in anxiety and depression scores are significantly greater than those of the control group. This result indicates that compared with routine pregnancy health education, PBL pregnancy health education based on the Omaha System adopted in this study has better intervention effects on the anxiety and depression of primiparas. This result is consistent with the previous research result that PBL pregnancy health education can effectively reduce postpartum depression and anxiety (Qiu et al. 2017).

There are mainly the following reasons why the health education model constructed in this study can relieve the anxiety and depression of primiparas in the observation group: on the one hand, PBL health education intervention reduces the fear in primiparas caused by their lack of delivery experience through helping them master relevant knowledge during pregnancy, enhance their sense of self-control and self-efficacy, and thereby improve their sensitivity to positive emotions (Mesurado et al. 2018). On the other hand, PBL health education, which is problem-oriented and patient-centered, provides pregnant women with psychological counseling, eases their psychological burden and alleviates their negative emotions such as anxiety and depression (Zhang et al. 2017). In this study, the existing problems in primiparas were classified and summarized from the four aspects including health, physiology, psychology and environment according to the Omaha System. On this basis, relevant nursing improvement measures were searched, selected and sorted out to formulate a syste- matic nursing intervention scheme. Then, health education intervention was carried out to increase pregnant women's understanding of postpartum emotional disorders and boost their ability of self-care and neonatal feeding. Upon timely identification of their negative emotions, guidance, counseling and mitigation programs were given instantly to effectively prevent genitive emotions in primiparas, help them maintain a happy mood and assist them to calmly deal with postpartum rehabilitation and neonatal feeding. Continuous efforts were made to improve and enrich nursing measures, with the aim to reduce anxiety and depression in primiparas.

Self-management refers to the ability of pregnant women to consciously control and supervise their thoughts and behaviors by relying on their own subjective initiative. This kind of self-monitoring allows pregnant women to maintain their own health and reinforce their adaptability. In this sense, it is a selfmonitoring behavior that can improve clinical results and reduce treatment costs (Soleiman et al. 2015). As shown in the results of this study, there is no significant difference between the observation group and the control group in self-management ability before the intervention. After a period of intervention (an unknown specific duration of intervention), there are significant increases in the self-management ability of primiparas in both groups, but the changes of the observation group in self-management ability and its four dimensions are significantly higher than those of the control group. This result suggests that compared with PBL health education, which has been found in some studies to be effective in improving the self-management ability of primiparas, PBL health education based on the Omaha System performs better in improving the self-management ability of primiparas (He \& Mu 2019).

According to previous studies, conventional health education is not targeted, but mostly focuses on unilateral knowledge transfer. Primiparas passively accept education, but have no way to solve their own problems and doubts, which is not conducive to health promotion during pregnancy and make it impossible to achieve "patient-centered" education in the process of implementation. Moreover, the rigid model of health education exacerbates the difficulty for patients to understand and cooperate (Yao et al. 2018). The PBL health education model proposed in this study can be "patientcentered" since it is a problem-oriented teaching model. Health education is carried out through medical workers' interaction with primiparas after helping them clarify their own problems, thus alleviating their psychological burden, enhancing their confidence, and increasing their self-management ability (Aydemir \& Onan 2015). Secondly, this study was conducted based on the Omaha System. According to some relevant surveys, most primiparas do not know much about their own diseases and treatments. Through the Omaha System, a comprehensive nursing practice and classification system, it is possible to not only instruct primiparas with pregnancy-related knowledge, but also aim to solve their 
specific problems in accordance with their actual situation. On the basis of enhancing their sense of participation, primiparas can be guided to actively think about themselves and have the participation of their family members or caregivers. In the whole delivery process, scientific knowledge and practical solutions are provided for primiparas to handle specific problems such as diet, cleaning, puerperal maintenance and newborn feeding. They are encouraged to acquire the ability to solve problems, give full play to their subjective initiative, and then improve their self-management ability. Besides, under this primipara-centered health education model, primiparas may perceive more social support. It has been found in some studies the perception of more social support can effectively improve the self-management ability of primiparas to a certain extent ( $\mathrm{Li}$ et al. 2015).

According to the results of this study, there is no significant difference between the observation group and the control group in quality of life before the intervention. After a period of intervention, there are increases in the quality of life of primiparas in both groups, but the changes of the observation group in quality of life and its four dimensions are significantly higher than those of the control group. This indicates that compared with routine pregnancy health education, PBL pregnancy health education based on the Omaha System has a better intervention effect on the quality of life of primiparas. This result is consistent with the previous research result that PBL health education can improve the quality of life of primiparas (Chen et al. 2019, Chen et al. 2020). Noticeably, Chen et al. (2019) intervened in primiparas through PBL health education, finding that their score in social relations, a dimension of quality of life, was $74.24 \pm 7.19$. In contrast, this score reached $75.22 \pm 8.2$ in this study of PBL health based on Omaha System, suggesting a more satisfactory effect than the effect of single PBL health education. The main reasons can be explained from the following aspects: the observation group adopted the nursing model based on the Omaha System. The follow-up of patients was conducted from the four directions, including health behavior, physiology, social psychology and environment. The follow-up results were used to summarize the corresponding problems. Meanwhile, the timely discovery of patients' social and psychological problems and the implementation of targeted nursing measures for patients contributed to the promotion of their rehabilitation and the increase in the score of their social relations. Chen et al. (2020) found that the quality of life of pregnant women accepting routine health education decrease significantly when delivery is approaching. In this study, there is no significant difference in the quality of life of pregnant women accepting health education based on the Omaha System near delivery and at admission. It can be seen that the model constructed in this study can alleviate some physiological or psychological discomfort arising in primiparas before delivery and thereby maintain their quality of life.
Childbirth definitely brings great changes to the life of primiparas. Firstly, primiparas often suffer poor sleep quality, fatigue, discomfort, obesity, insufficient breastfeeding and other manifestations before or after delivery (Otero-Naveiro et al. 2021). Secondly, in the special process of pregnancy and delivery, pregnant women are forced to leave their jobs for a period of time, probably having lower self-efficacy. Thirdly, long-term stay at home and storage of interpersonal communication with the outside world tend to lead to depression, anxiety and other adverse emotions in pregnant women (Phua et al. 2020). Clinical evidence shows that routine nursing focuses on the treatment of patients' diseases and their rehabilitation, and therefore can only meet the physiological and safety needs of patients. However, with the continuous improvement of medical models in recent years, more and more attention has been paid to patients' demand for psychological nursing (Shen et al. 2020). In the process of pregnancy and delivery, the changes of hormones in the body may trigger the incidence of depression and anxiety, resulting in the failure to meet their safety needs. Moreover, due to the particularity of pregnancy, pregnant women need to leave their jobs and even to stay in bed for a period of time, thus making it difficult to meet their interpersonal needs and undermining their self-realization to a certain extent. All these problems faced by primiparas are the drivers of the decline in their quality of life.

PBL pregnancy education was conducted in this study based on the Omaha System to intervene in pregnant women. The detailed contents of this model include full consideration of the actual problems faced by primiparas, comprehensive analysis of the various needs of pregnant women, attention to their physiological needs in the initial stage, followed by gradual assistance to solve their psychological and emotional needs to achieve better nursing effect. Zhang et al. (2018) transfer of correct healthy puerperal maintenance knowledge to pregnant women, and guidance for primiparas to establish a scientific lifestyle and avoid possible problems such as obesity, fatigue and insufficient breastfeeding in real life. On the premise of the building of a favorably trustful doctor-patient relationship, it is also necessary to encourage pregnant women to actively express their needs, correctly vent their negative emotions and seek targeted training such as yoga and music therapy in accordance with their actual needs to alleviate their negative emotions (Rong et al. 2021). While solving the emotional problems of pregnant women, nursing workers would should communicate more with their family members, call on them to pay attention to and accompany their pregnant women, assist their family members to create a safe and loving living environment for pregnant women, keep pregnant woman in a good state of mind, and reduce their psychological pressure. The quality of life of primiparas can thereby be improved when they have their various needs met, perceive more social support and recover the optimal normal physiological and psychological state. 


\section{CONCLUSION}

PBL pregnancy health education was conducted in this study based on the Omaha System to intervene in 164 primiparas. The results show that this new nursing model can effectively improve the self-management ability and quality of life of primiparas, reduce their anxiety and depression to a large extent, significantly outperform conventional pregnancy health education in nursing, and therefore is highly applicable in obstetric nursing. At the same time, the results of the study also suggest that PBL pregnancy health education based on the Omaha System can help primiparas master more delivery knowledge and improve their overall health level.

\section{Acknowledgements: None.}

\section{Conflict of interest: None to declare.}

\section{Contribution of individual authors:}

All authors made equal contributions

\section{References}

1. Aydemir S, Onan N: The Relationship Between Maternal Self-confidence and Postpartum Depression in Primipara Mothers: A Follow-Up Study. Community mental health journal 2020; 56(8): 1-8

2. Ayele TA, Azale T, Alemu $K$, et al: Prevalence and associated factors of antenatal depression among women attending antenatal care service at Gondar University Hospital, Northwest Ethiopia. PloS one 2016; 11(5): e0155125

3. Beňová E, Boledovičová $M$, Bowles KH: Problems, interventions and outcomes of the Omaha system in nursing clinical practice. Kontakt 2016; 18(3): e164-e169

4. Chen L, Wu JY, Tang HJ: Application effect of nursing intervention mode guided by Omaha system in preventing postpartum depression. China Modern Medicine 2020; 27(35): 230-2

5. Chen YM, Xing YY, He XY: Effect of problem-oriented health education on negative emotions and quality of life in cesarean section. Capital Medicine 2019; 26(14): 132-3

6. Coll CDVN, da Silveira MF, Bassani DG, et al: Antenatal depressive symptoms among pregnant women: Evidence from a Southern Brazilian population-based cohort study. Journal of Affective Disorders 2017; 209: 140-6

7. He XL, Mu NH: Effect of problem-Based education model on self-management ability and maternal as well as Infant complications in patients with gestational diabetes mellitus. Journal of practical clinical medicine 2019; 23(15): 74-6

8. Kearney BY, Fleischer BJ: Development of an Instrument to Measure Exercise of Self-care Agency. Research in Nursing \& Health 1979; 2(1): 25-34

9. Li CY, Zhang CL, Zhang FP: Effect of nursing intervention with comprehensive social support on self-management ability and adverse pregnancy outcome of primipara. Laboratory Medicine and Clinic 2019; 16:3197-9
10. Li Y, Zeng Y, Zhu W, et al: Path model of antenatal stress and depressive symptoms among Chinese primipara in late pregnancy. BMC pregnancy and childbirth 2016; 16:1-7

11. Martin KS, Monsen KA , Bowles KH: The Omaha system and meaningful use: applications for practice, education, and research. Comput Inform Nurs 2011; 29(1): 52-8

12. Maxim T, Nadya G, Bowles KH: The Omaha System: a systematic review of the recent literature. Journal of the American Medical Informatics Association Jamia 2014; 21(1): $163-70$

13. McElroy E, Fearon P, Belsky J, et al: Networks of depression and anxiety symptoms across development. Am Acad Child Adolesc Psychiatry 2018; 57(12): 964-73

14. Melo F, Arzeno FY: Transcranial magnetic stimulation for treatment of major depression during pregnancy: a review. Trends in Psychiatry \& Psychotherapy 2016; 38(4):190-7

15. Mesurado B, Vidal EM, Mestre AL: Negative emotions and behaviour: The role of regulatory emotional selfefficacy. Journal of Adolescence 2018, 64: 62-71

16. Mossie TB, Sibhatu AK, Dargie A, et al: Prevalence of antenatal depressive symptoms and associated factors among pregnant women in Maichew, North Ethiopia: an institution based study. Ethiopian journal of health sciences 2017; 27: 59-66

17. Nakamura Y, Okada T, Morikawa M, et al: Perinatal depression and anxiety of primipara is higher than that of multipara in Japanese women. Scientific Reports 2020; 10:1-10

18. Otero-Naveiro A, Gómez-Fernández C, Álvarez-Fernández $R$, et al: Maternal and fetal outcomes during pregnancy and puerperium in obese and overweight pregnant women. A cohort study. Archives of Gynecology and Obstetrics 2021; 1-8, Online first

19. Phua DY, Chen H, Chong YS, et al: Network Analyses of Maternal Pre-and Post-Partum Symptoms of Depression and Anxiety. Frontiers in Psychiatry 2020;11: 785

20. Qiu XT, Huang HQ, Lu SX, et al: Study on Influence of PBL-type Health Education During Pregnancy on Postpartum Emotional State and Parenting Self-efficacy of Delivery Women. Medical Innovation of China 2017; 14(17): 90-3

21. Lu X, Liu L, \& Yuan R: Effect of the information support method combined with yoga exercise on the depression, anxiety, and sleep quality of menopausal women. Psychiatria Danubina 2020; 32(3-4): 380-388

22. Rong L, Wang R, Ouyang Y Q, et al: Efficacy of yoga on physiological and psychological discomforts and delivery outcomes in Chinese primiparas. Complementary Therapies in Clinical Practice 2021; 44: 101434

23. Sartorius N, Üstün TB, Lecrubier $Y$, et al: Depression comorbid with anxiety: Results from the who study on psychological disorders in primary health care. $\mathrm{Br} J$ Psychiatry 1996; 168(S30): 38-43

24. Seda Ö, Erkek ZY: The Relationship Between Pregnant Women'Emotional Intelligence and Fear of Childbirth, Readiness For Childbirth and Ways of Coping with Stress. Journal of Basic and Clinical Health Sciences 2021; 5(2): 83-93

25. Shen YY, Sheng LL, Yan M, et al: Effect of comprehensive nursing based on Maslow's hierarchy of needs theory on psychological stress, functional rehabilitation and quality of life of postoperative breast cancer patients. International Journal of Nursing 2020; 39(17): 3149-52 
26. Shi YJ, Liu H, Feng YL, et al: Analysis of pregnancy related anxiety and it influencing factors in late pregnancy women in Wuxi City. Chinese nursing research 2019; 33(1): $87-91$

27. Skouteris H, Wertheim EH, Rallis S, et al: Depression and anxiety through pregnancy and the early postpartum: An examination of prospective relationships. Affect Disord 2009; 113(3): 303-8

28. Soleiman EY, Majlessi F, RahimI FA: Measurement of the constructs of health belief model related to self-care during pregnancy in women referred to South Tehran health network. Journal of Community Health 2015; 1:89-98

29. Su J: Effect of prenatal PBL health education on disease knowledge and self-efficacy of patients with gestational diabetes mellitus. Nursing Practice and Research 2016; 13:4-6

30. Wang LK: Application of health education during pregnancy in obstetrics and Gynecology Nursing. China Health Care \& Nutrition 2019; 29(4): 204-5

31. Wang QQ, Wang JH: Validity and reliability of whoqolbref scale among nurses with night shift. Chinese Journal of Public Health 2013; 29(1):111-4

32. Wang $Y B, X u L Y$, Yang $L S$, et al: Reliability and validity of Chinese version of the exercise of self-care agency scale in schizophrenia patients. Journal of Clinical Psychiatry 2014; 24(2): 104-7

33. Woody CA, Ferrari AJ, Siskind DJ, et al: A systematic review and meta-regression of the prevalence and incidence of perinatal depression. Journal of Affective Disorders 2017; 219:86-92

34. Xiao H: Application value of Omaha System holistic nursing scheme in patients with acute attack of chronic obstructive pulmonary disease.Chronic Pathematology Journal 2018; 19(9): 149-150

35. Yao LP, Li BH, Guo JB, et al: Practice of health education using the teach - back method for patients with gestational diabetes mellitus. Journal of Nursing Science 2018; 33(10): 9-12

36. Ye $Q H$, Chen $Z B$, Tang $K$ : Research progress of postpartum depression. Hainan Medical Journal 2016; 27(20): 3376-8

37. Zhang XX, Zhao F, Chang $Y$, et al: Effect of probLemoriented heaLth education on postpartum depression and hormone LeveLs in primipara. Chinese Journal of Practical Nursing 2019; 35(10): 784-789

38. Zhang LL, Yin F, Zhou LQ: Effect of Maslow's hierarchy of needs nursing model on postoperative treatment compliance and quality of life of cervical spondylosis. Laboratory Medicine and Clinic 2018; 15(9): 132-4

39. Zhao H, Jing T: Effect of nursing intervention on prevention of postpartum depression.Shanxi Medical Journal 2019; 48(17): 94-96

40. Zung $W W$ : A rating instrument for anxiety disorders. Psychosomatics 1971; 12(6): 371-379

41. Zung WW: A self-rating depression scale. Archives of General Psychiatry 1965; 12:63-70

Correspondence:

Qiong Pan

Yunnan Xinxing Occupations Institute,

Shuihaizi, national economic and Technological Development Zone

Kunming, 650217, Yunnan, China

E-mail: panqiong_8866@163.com 